\title{
Análise de metais no sedimento na baía do Guajará Belém/PA
}

É importante manter a qualidade dos corpos hídricos, já que o mesmo exerce um significativo papel para o ecossistema aquático e população. Na baía do Guajará em Belém-PA, não é diferente, a mesma desempenha importante papel para os organismos aquáticos e exerce uma considerável influência econômica para o estado. Entretanto, sua qualidade está sendo prejudicada devido as mudanças provocadas por atividades naturais e/ou antrópica. A avaliação ambiental por meio do estudo de sedimentos (fundo e suspensão) vem se destacando como um meio de avaliar as condições ambientais de um corpo hídrico, pois desempenha o papel de acumular, reprocessar e transferir os elementos químicos presentes nesse recurso natural, permitindo também quantificar o grau de poluição de um determinado local e verificar possíveis contaminações que a água e os organismos aquáticos podem estar sujeitos ao logo do tempo. permitindo também quantificar o grau de poluição de um determinado local e verificar possíveis contaminaçães que a água e os organismos aquáticos podem estar sujeitos ao logo do tempo. É necessário a realização de estudos detalhados dos sedimentos de rios, a fim de saber as origens e o nível de contaminação que o ambiente está sendo afetado. Dessa forma o estudo analisou os metais $\mathrm{Cr}$, Cd, Cu e Fe no sedimento de fundo, na forma total e lixiviado na baia do Guajará, Belém/PA, calculando o FC, Igeo e FE, no intuito de verificar o grau de contaminação 2015 e 2016, em períodos sazonais distintos (chuvoso e menos chuvoso). As amostras foram coletadas com dragas do tipo Van Veen. Foi realizado o processo de digestão ácida e lixiviação do sedimento coletado. Os metais foram quantificados através da técnica de Espectrometria de Emissão Ótica com Plasma Acoplado Induzido (ICP OES). Na análise do sedimento total, o Cd apresentou valores acima do permitido por PEL e CONAMA no 454/2012, já na fase lixiviada, todos os metais ficaram dentro do permitido pela legislação. No geral, os pontos apresentaram de baixa à moderada contaminação, caracterizados como ambiente não poluído à moderadamente poluído e com deficiência de enriquecimento. É necessário o monitoramento contínuo da baía do Guajará, haja vista que a concentração de contaminantes no sedimento pode atingir valores que causam prejuízos para biota aquática, e isso também coloca em risco a saúde da população humana, considerando que o processo de contaminação pode começar no sedimento e atingir os níveis mais altos da cadeia alimentar.

Palavras-chave: Sedimento; Metais; Contaminação; Diagnóstico Ambiental.

\section{Analysis of metals in the sediment in the bay of Guajará Belém/PA}

It is important to maintain the quality of the water bodies, since it plays a significant role for the aquatic ecosystem and population. In the bay of Guajará in Belém-PA, it is no different, it plays an important role for aquatic organisms and exerts a considerable economic influence on the state. However, its quality is being impaired due to the changes caused by natural and / or anthropic activities. Environmental assessment through sediment studies (bottom and suspension) has been highlighted as a means to evaluate the environmental conditions of a wate body, since it plays the role of accumulating, reprocessing and transferring the chemical elements present in this natural resource, quantify the degree of pollution of a given site and check for possible contamination of water and aquatic organisms with time. It is necessary to carry out detailed studies of river sediments in order to know the origins and the level of contamination that the environment is being affected. Thus, the study analyzed the $\mathrm{Cr}, \mathrm{Cd}, \mathrm{Cu}$ and Fe metals in the bottom sediment, in the total form and leached in Guajará bay, Belém / PA, calculating the $\mathrm{FC}$, Igeo and FE, in order to verify the degree of contamination of the sediment, the origin of this pollution, and thus obtain a diagnosis of the research site. The study was performed in 4 points (PT01, PT02, PT03 and PT04). The collections occur in the year 2015 and 2016, in distinct seasonal periods (rainy and less rainy). The samples were collected with Van Veen dredges. The process of acid digestion and leaching of the collected sediment was carried out. The metals were quantified by the technique of Optical Emission Spectrometry with Induced Coupled The process of acid digestion and leaching of the collected sediment was carried out. The metals were quantified by the technique of Optical Emission Spectrometry with Induced Coupled
Plasma (ICP OES). In the analysis of the total sediment, the Cd presented values above that allowed by PEL and CONAMA no $454 / 2012$, already in the leached phase, all metals were within the allowed by the legislation. In general, the points presented low to moderate contamination, characterized as an unpolluted environment to a moderately polluted and enriched deficiency. Continuous monitoring of the Guajará Bay is necessary since the concentration of contaminants in the sediment can reach values that cause damage to aquatic biota, and this also endangers the health of the human population, considering that the contamination process can begin in the sediment and reach the highest levels of the food chain.

Keywords: Sediment; Metals; Contamination; Environmental Diagnosis.

Topic: Uso de Recursos Naturais

Reviewed anonymously in the process of blind peer
Received: 10/04/2018

Approved: 24/05/2018
Letícia Furtado dos Santos

Universidade Federal do Pará, Brasil

http://lattes.cnpq.br/0057907745892434

leticiafurtadosantos@gmail.com

Adriano Marlisom Leão de Sousa

Universidade Federal Rural da Amazônia, Brasil

http://lattes.cnpq.br/4371199443425884

marlisoms@yahoo.com.br

Kelson do Carmo Freitas Faial (ic

Instituto Evandro Chagas, Brasil

http://lattes.cnpq.br/5038449535463689

http://orcid.org/0000-0001-7094-4902

kelsonfaial@iec.gov.br
Referencing this:

SANTOS, L. F.; SOUSA, A. M. L.; FAIAL, K. C. F.. Análise de metais no sedimento na baía do Guajará Belém/PA. Revista Ibero Americana de Ciências Ambientais, v.9, n.4, p.314-325, 2018. DOI:

http://doi.org/10.6008/CBPC2179-6858.2018.004.0025 


\section{INTRODUÇÃO}

Manter a qualidade dos corpos hídricos e utilizar o sistema aquático de forma sustentável vem sendo um dos grandes desafios atualmente. Essa qualidade é influída por diversos fatores, como a cobertura vegetal, topografia, geologia e uso e manejo do solo. Esses fatores influenciam nas condições físicas, químicas e biológicas de uma bacia hidrográfica. Entretanto, com as alterações provocadas pelas atividades antrópicas, esses conjuntos podem sofrer alterações e ocasionar perturbações ao ecossistema aquático (FURLAN et al., 2009).

Devido a capacidade do sedimento em acumular compostos em seu compartimento, o mesmo vem se destacando como um meio de avaliar as condições ambientais de um corpo hídrico. O sedimento é caracterizado como material sólido ou semissólido que é depositado no fundo de rios, lagos e reservatórios e conduzido a partir da interação constante e contínua dos processos de intemperismo e erosão (LICHT, 1998).

Nos ecossistemas aquáticos, o sedimento desempenha o papel de acumular, reprocessar e transferir metais. Tais elementos químicos ficam potencialmente disponíveis para os organismos aquáticos e podem ser liberados através da atividade microbiana e mudanças nos vários parâmetros físico-químicos que afetam o meio, como o pH, salinidade e condições de óxido-redução (MORREIRA et al., 2003). A avaliação de metais no sedimento, é importante devido essa matriz ser um compartimento que funciona como um sistema de estoque de poluentes, devido sua alta capacidade de adsorção de elementos químicos (TRINDADE et al., 2012).

Existe os metais que não ocasionam prejuízos para o meio, porém com seu acumulo, podem trazer malefícios para biota aquática, como por exemplo o Fe (ferro), que pode se concentrar em valores maiores em ambientes que possui intensa atividade portuária. Há também os metais pesados, como o $\mathrm{Cr}$ (cromo), $\mathrm{Cd}$ (cádmio) e Cu (cobre), que em pequena quantidade já acarretam danos para o ecossistema aquático e sociedade (MALDONADO et al., 2009).

A análise de metais no sedimento possibilita quantificar o grau de poluição de um determinado lugar e verifica possíveis contaminações que a água e os organismos aquáticos podem estar sujeitos ao logo do tempo. Para obter tais informações, utiliza-se ferramentas de avaliação, dentre as quais se destacam o Fator de Contaminação (FC), o Índice de Geoacumulação ( $\mathrm{I}_{\mathrm{geo}}$ ) e o Fator de Enriquecimento (FE). Essas ferramentas têm por objetivo estimar o risco que pode afetar a saúde dos ecossistemas aquáticos e da população humana (BELO et al., 2010).

O FC faz uma estimativa geral da qualidade do sedimento, já o $I_{\text {geo }}$ possibilita obter dados quantitativos sobre a intensidade da contaminação, no qual é classificado por nível de poluição. Já o FE avalia o grau de influência antrópica que o ambiente pode estar sofrendo, o mesmo diferencia o enriquecimento originado das atividades antrópicas e aqueles acarretadas por fenômenos naturais (SANTOS et al., 2012; ARAÚJO et al., 2012; SILVA et al., 2002). Assim, é de grande importância a realização de estudos detalhados 
dos sedimentos de rios, a fim de saber as origens e o nível de contaminação que o ambiente está sendo afetado.

Se o sedimento estiver contaminado por poluentes, o mesmo dependendo das condições físicoquímica da água pode ser biodisponibilizado para a coluna d'água e para os organismos que estão no 1o nível trófico da cadeia alimentar, como as algas e os macro invertebrados bentônicos, tais indivíduos estão na base da teia alimentar e servem de alimento para a biota aquática, como os peixes, que são em sua maior parte ingeridos pela população humana. Dessa forma, ressaltasse a necessidade do monitoramento frequente da baía de Guajará, já que a mesma exerce um importante papel para a biota aquática, população e para a economia do Pará (SILVA et al., 2008; REPULA et al., 2012).

À vista disso, esta pesquisa avaliou os metais cádmio $(\mathrm{Cd})$, cromo $(\mathrm{Cr})$, cobre $(\mathrm{Cu})$ e ferro $(\mathrm{Fe})$ no sedimento total e na lixiviação desse sedimento na baía do Guajará, localizada na cidade de Belém no Estado do Pará, analisando o fator de contaminação $(F C)$, o índice de geoacumulação ( Igeo $_{\text {ge }}$ o fator de enriquecimento (FE), a fim verificar o grau de contaminação do sedimento e a origem dessa poluição, e assim obter um diagnóstico do local de estudo.

\section{METODOLOGIA}

\section{Área de estudo}

A cidade de Belém, localizada no estado do Pará, é um município que possui uma extensão territorial de 1.059,458 km², com população estimada em 1.452 .275 pessoas, com densidade demográfica de 1.315,26 $\mathrm{hab} / \mathrm{km}^{2}$ e IDH de 0,746. A mesma exerce um importante papel para a população e economia do estado, suas principais atividades econômicas envolvem o uso de recursos naturais.

No entorno de Belém encontra-se a baía do Guajará, posicionada na margem direita do Rio Pará

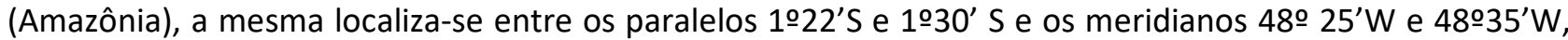
com cerca de $30 \mathrm{~km}$ de extensão e $4 \mathrm{~km}$ de largura, e recebe a contribuição hídrica e sedimentar de dois sistemas fluviais: rio Guamá e rio Acará. A deposição e dispersão do sedimento na baía é principalmente controlada pela baixa topografia, pelo grande aporte fluvial e atuação das correntes de maré (GREGÓRIO et al., 2009; MIRANDA et al., 2007). Essa região exerce grande importância econômica para o estado do Pará, já que na sua margem direita ocorrem intensas atividades portuárias, de transporte e armazenamento de petróleo, algo que movimenta a economia da região (MIRANDA et al., 2007). O estudo foi realizado nos seguintes pontos da baía de Guajará (figura 1):

Porto Bom Jesus (PT01): (S01²8'41.8' W048²9'19.1'), região portuária que está localizado na jusante do canal Quintino Bocaiuva, região na qual ocorre a drenagem de efluentes líquidos que passa por alguns bairros da periferia de Belém (figura 2).

Porto Belém - Armazém três Companhia Docas do Pará (PT02): (S01²6’58.0" W048³0'05.6”) localizado a jusante do mercado do Ver-o-Peso e a montante da Base Naval de Val-de-cães a uma distância 
de $120 \mathrm{~km}$ do oceano Atlântico. Nesse ponto ocorre intenso tráfico de embarcação por conta de atividades portuária (figura 3).

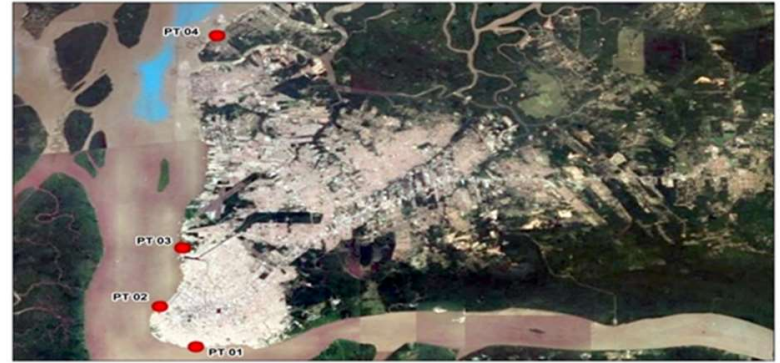

Figura 1: PT 01, Porto Bom Jesus; PT 02, Porto BelémArmazém três Companhia Docas do Pará; PT 03, Canal São Joaquim; PT 04, Outeiro. Fonte: Google Earth, 2017.

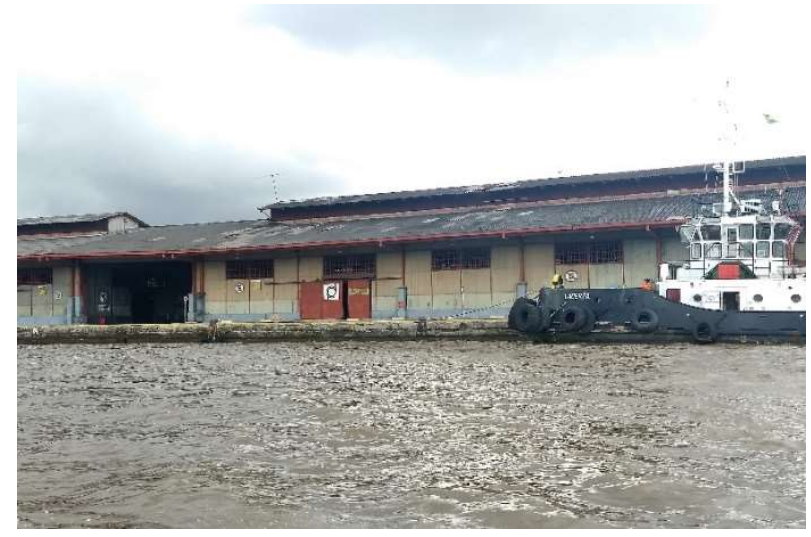

Figura 3: Ponto 02, Porto Belém-Armazém três Companhia Docas do Pará.

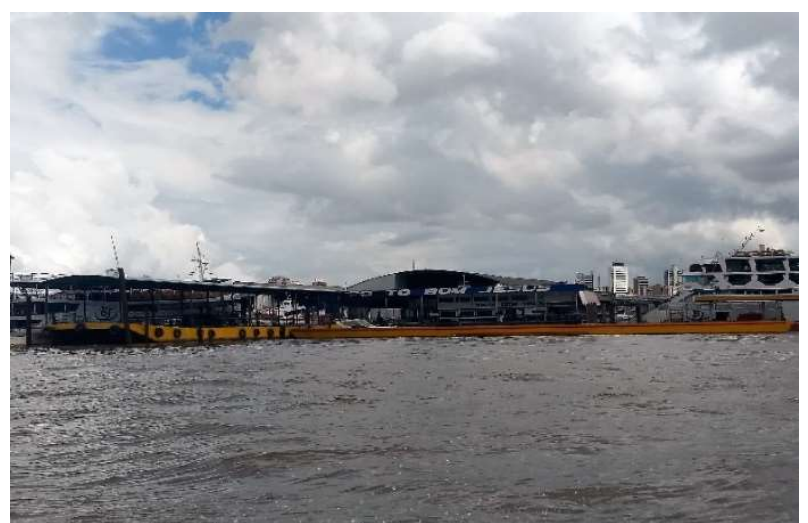

Figura 2: Ponto 01, Porto Bom Jesus.

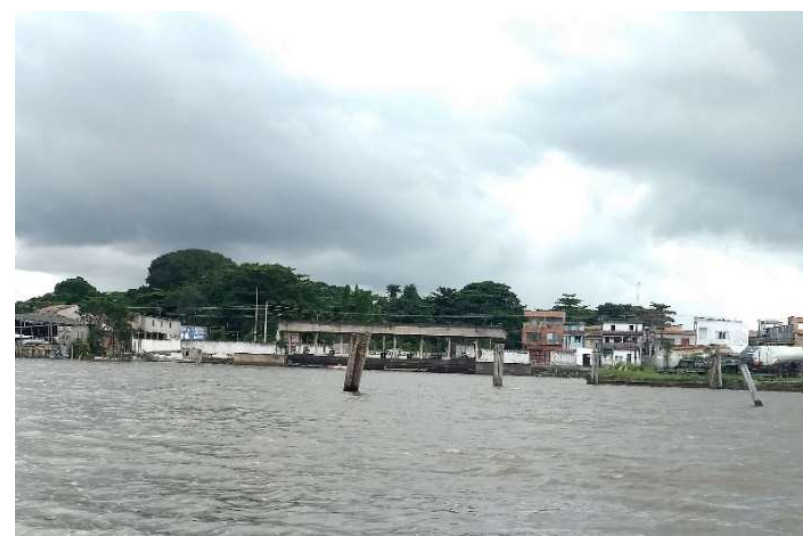

Figura 4: Ponto 03, Canal São Joaquim.

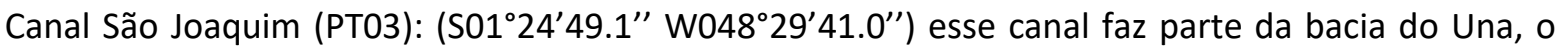
canal do Una é um igarapé que recebe $40 \%$ do esgoto urbano de Belém que é lançado sem pré-tratamento e, além disso, fica próximo ao terminal de Miramar, local em que ocorre intensa movimentação de combustíveis líquidos e gasosos (figura 4).

Outeiro (PT04): (S01¹6'16.3” W048²8'59.9”) localizado próximo praia da Brasília, ambiente no qual recebe frequentemente os banhistas, nesse ponto de estudo há uma ponte inativa de embarcações, entretanto, esporadicamente a mesma ainda recebe alguns navios. Seu acesso fluvio-marítimo vai da foz do rio Pará, que deságua no Atlântico, e desce através do Canal do Mosqueiro até a baía de Guajará no qual se situa esse ponto (figura 5).

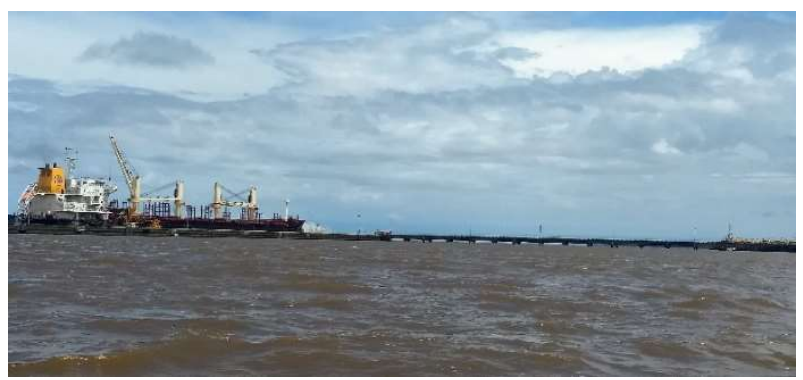

Figura 5: Ponto 04, Outeiro. 


\section{Coleta}

As coletas ocorreram durante marés de sizígias (marés que ocorrem nas luas novas e cheias, produzindo as maiores marés altas e as menores marés baixas) (MIGUENS, 1996), durante os seguintes meses e anos: Março-2015, Junho-2015, Setembro-2015, Dezembro 2015, Março-2016, Junho-2016, correspondente aos períodos chuvoso e menos chuvoso da região amazônica. O sedimento de fundo foi coletado com o auxílio de draga do tipo Van Veen $(20 \times 20 \times 20 \mathrm{~cm})$ (Figura 6) de acordo com o procedimento de coletas proposto por Eleftheriou et al. (2005), foi retirado aproximadamente $1 \mathrm{Kg}$ de amostra cada ponto analisado. Após coletadas as amostras foram acondicionadas em sacos plásticos, mantidas sob refrigeração e levadas ao laboratório de Toxicologia, na Seção de Meio Ambiente do Instituto Evandro Chagas (SAMAM/IEC).

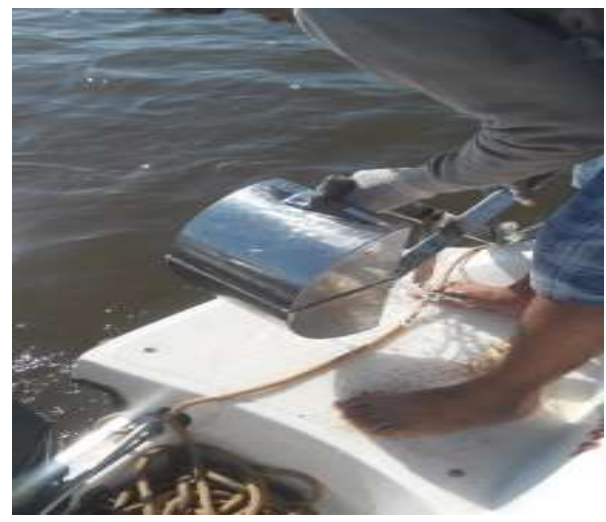

Figura 6: Coleta do sedimento de fundo.

\section{Análise Laboratorial}

\section{Sedimento de fundo}

As amostras passaram pelo processo de secagem a temperatura ambiente, retirando-se após esse período, a fração bruta e em seguida foi realizado o processo de maceramento e peneiração em uma malha de $53 \mathrm{~mm}$ e assim tirado a fração de 270 mesh.

\section{Digestão das amostras de sedimento}

$\mathrm{Na}$ digestão das amostras de sedimento foi utilizada uma massa aproximada de 0,2g. As amostras foram acidificadas com $3 \mathrm{ml}$ de $\mathrm{HNO}_{3}$ (ácido nítrico, 65\% v.v ${ }^{-1}$ ), $1 \mathrm{ml} \mathrm{HCl}$ (ácido clorídrico 38\% v.v ${ }^{-1}$ ), $1 \mathrm{ml}$ de

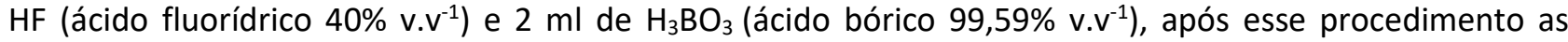
amostras foram expostas à radiação por micro-ondas (MarsXpress - CEM) para digestão total dos elementos.

Foi utilizado o programa com rampa de potência e temperatura detalhadas na tabela 1, em seguida as amostras foram retiradas do forno e aferidas com água deionizada para um volume final de $50 \mathrm{ml}$.

Tabela 1: Programação da rampa de temperatura do forno de micro-ondas.

\begin{tabular}{|l|l|l|l|l|}
\hline Rampa & Potência $(\mathrm{W})$ & Tempo (minutos) & Temperatura $\left({ }^{\circ} \mathrm{C}\right)$ & Estabilização (minutos) \\
\hline $\mathbf{1}$ & 400 & 2 & 110 & 1 \\
\hline $\mathbf{2}$ & 800 & 4 & 150 & 1 \\
\hline $\mathbf{3}$ & 800 & 30 & 200 & 1 \\
\hline $\mathbf{4}$ & 800 & 10 & Resfriamento & 0 \\
\hline
\end{tabular}




\section{Lixiviação do sedimento}

Para a determinação dos metais biodisponíveis, pesou-se 1,0g da amostra de sedimento em um Erlenmeyer e foi adicionado $25 \mathrm{ml}$ de $\mathrm{HCl}$ (ácido clorídrico) a 0,10 mol L-1 , passando pelo processo de agitação em um agitador horizontal (RECIPRO SHAKER, SR-1) a 200 rpm, por 2 h. Após esse processo as amostras foram centrifugadas (COTTA, et al., 2006).

\section{Determinação dos metais nas amostras de sedimento de fundo.}

Os metais foram quantificados pela técnica de Espectrometria de Emissão Ótica com Plasma Acoplado Induzido (ICP OES), modelo Vista- MPX CCD simultâneo (Varian, Mulgrave, Austrália), configuração axial e equipado com um sistema de amostragem automático (SPS-5). O controle das condições operacionais do ICP OES foi realizada com o sofware ICPExpert Vista.

\section{Normalização dos dados}

A normalização é um procedimento utilizado para obter e quantificar concentrações irregulares de metais em sedimentos (HORTELLANI et al., 2008). Nesse trabalho foi utilizado a normalização através da extrapolação da curva de regressão, no qual os pontos do estudo que ficaram fora do limite de confiança da regressão (intervalo de 95\%) não foram utilizados para obtenção do valor de referência para o metal analisado.

Como elemento normalizador da concentração dos metais em estudo no sedimento e como o elemento de referência natural (background), foi utilizado o teor do Al, devido o mesmo ser um dos mais abundantes na crosta terrestre e apresentar melhor recuperação na análise das amostras certificada, no processo de quantificação dos metais no sedimento.

\section{Fator de contaminação (FC)}

Para uma avaliação geral da qualidade do sedimento, foi utilizado o fator de contaminação (FC) proposto por Hakanson (1980) (equação 01). Esse fator é obtido através da seguinte equação:

$$
F C=\frac{C n}{C B n} \quad \text { Equação } 01
$$

Segundo Hakanson (1980), os resultados obtidos através do FC são classificados através da seguinte forma: FC< 1= Baixa contaminação; FC=1 a 3= Moderada contaminação; FC= 3 a 6= Considerável contaminação; $F C<6=$ Alta contaminação.

\section{Índice de Geoacumulação (Igeo)}

O I geo é realizado a fim de saber a intensidade da contaminação, o mesmo é calculado através da seguinte equação de Müller (1979) (equação 02): 


$$
I_{\text {geo }}=\log _{2}\left[\left(C_{n} / 1,5 \times C_{B n}\right)\right] \quad \text { Equação } 02
$$

Em que: $\mathrm{C}_{n}=$ Concentração do metal ' $\mathrm{n}$ ' na fração fina do sedimento; $\mathrm{C}_{\mathrm{Bn}}=$ Concentração média do metal ' $\mathrm{n}$ ' no background; 1,5 = Fator de correção para prováveis variações do 'background'.

De acordo com os valores que são obtidos por via do $\mathrm{I}_{\mathrm{GEO}}$, os fatores relacionados ao grau de poluição são distribuídos em 7 classes, conforme é apesentado na tabela 2.

Tabela 2: Classificação do grau de poluição através do Igeo.

\begin{tabular}{|l|l|l|}
\hline Classificação & Classe do I geo & I geo $_{\text {Média "Background" }}$ \\
\hline Extremamente Poluído & 6 & $>5$ \\
\hline Fortemente à extremamente Poluído & 5 & $>4$ a 5 \\
\hline Fortemente Poluído & 4 & $>3$ a 4 \\
\hline Moderadamente à Fortemente Poluído & 3 & $>2$ a 3 \\
\hline Moderadamente Poluído & 2 & $>1$ a 2 \\
\hline Não Poluído a Moderadamente poluído & 1 & $>0$ a 1 \\
\hline Praticamente não Poluído & 0 & $<0$ \\
\hline
\end{tabular}

Fonte: Müller (1979).

\section{Fator de Enriquecimento (FE)}

Esse fator de enriquecimento definido por Gresens é utilizado para avaliar o grau de impacto que o ambiente estar sofrendo, e designar a origem desse impacto, que pode ser de fonte natural ou antropogênica.

O FE é obtido através do seguinte cálculo utilizado por Yongming et al., 2006 (equação 03):

$\mathrm{FE}=\frac{(\mathrm{Cm} / \mathrm{Cvrl}) \mathrm{amostra}}{(\mathrm{Bm} / \mathrm{Bvrl}) \mathrm{VRL}} \quad$ Equação 03

Em que:

$\mathrm{Cm}=$ Concentração de determinado elemento no local de interesse;

Cvrl= Concentração do elemento de referência;

$\mathrm{Bm}=$ Valor de referência local do elemento em estudo;

Bvrl = Valor de referência local do elemento de referência.

A contaminação do ambiente calculada através do FE é classificada da seguinte forma: $\mathrm{FE}<2=$ Deficiência de enriquecimento; FE = 2 - 5 = Enriquecimento moderado; FE = 5 - 20 = Enriquecimento significativo; $\mathrm{FE}=20-40=$ Enriquecimento muito alto; $\mathrm{FE}>40=$ Enriquecimento extremamente alto.

Quando o valor do FE $\leq 2$ aponta para contribuição natural, porém quando ele apresentar FE > 2 é devido a contribuição de fonte antropogênica (ARAÚJO et al., 2012). Para o tratamento estatístico dos resultados obtidos na pesquisa, foi utilizado o software MINITAB 16.

\section{RESULTADOS}

Os resultados da análise dos metais no sedimento total e lixiviado foram comparados com os valores do controle de qualidade estabelecidos pela Resolução do Conselho Nacional do Meio Ambiente (CONAMA) no454 de 01 Novembro de 2012 (BRASIL, 2012) e pelo Conselho Canadense do Ministério do Meio Ambiente, através do TEL (Threshold Effect Level), que se refere ao nível de efeito limiar abaixo do qual não ocorre efeito prejudicial à comunidade biológica; e PEL (Probable Effect Level), com referência ao nível de efeito provável à ocorrência de efeitos antagônicos à biota (CCME, 1995). Dentre os metais analisados, não há valores de referência para o Fe nessas resoluções. As figuras 7, 8, 9 e 10 apresentam respectivamente as concentrações 
dos metais $\mathrm{Cd}, \mathrm{Cr}$, $\mathrm{Cu}$ e $\mathrm{Fe}$ no sedimento total, conforme os pontos e os períodos chuvoso ( $\mathrm{CH}$ ) e menos chuvoso $(\mathrm{MCH})$ da coleta.

Cd

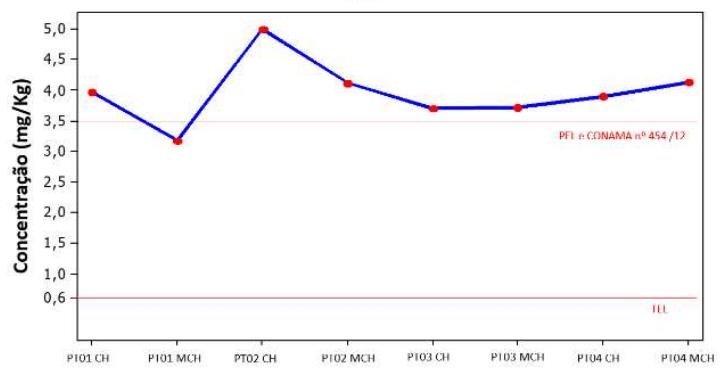

Figura 7: Concentração do $\mathrm{Cd}$ no sedimento em $\mathrm{mg} / \mathrm{Kg}$, na baía do Guajará, no período entre 2015 e 2016.

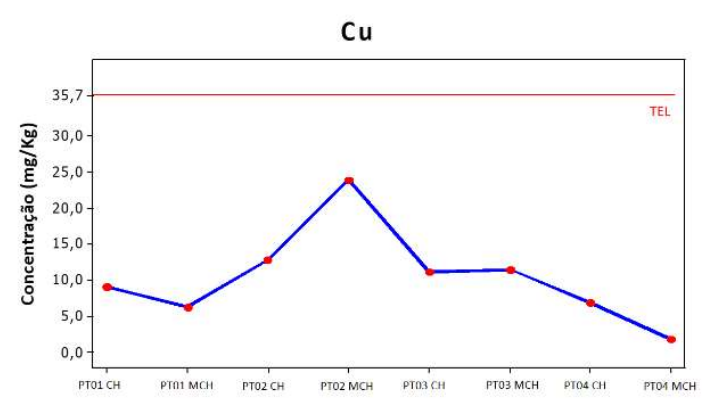

Figura 9: Concentração do Cu no sedimento em $\mathrm{mg} / \mathrm{Kg}$, na baía do Guajará, no período entre 2015 e 2016.

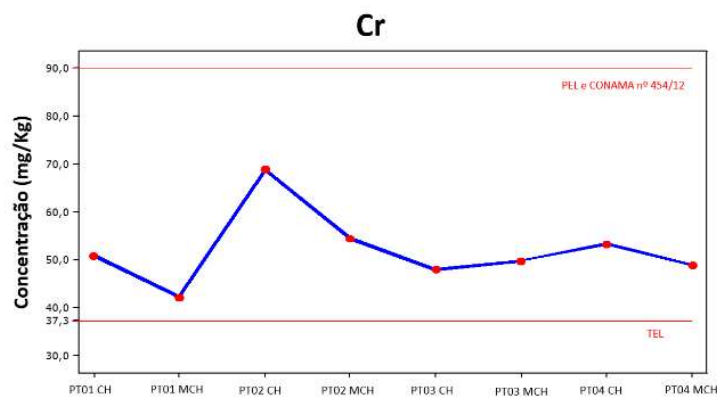

Figura 8: Concentração do $\mathrm{Cr}$ no sedimento em $\mathrm{mg} / \mathrm{Kg}$, na baía do Guajará, no período entre 2015 e 2016.

Fe

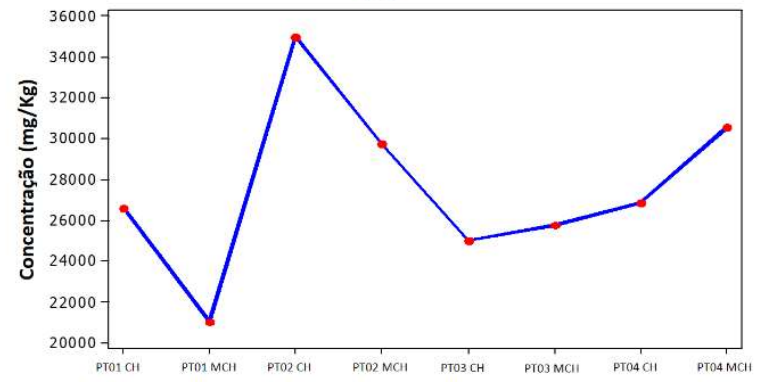

Figura 10: Concentração do Fe no sedimento em $\mathrm{mg} / \mathrm{Kg}$, na baía do Guajará, no período entre 2015 e 2016.

Tabela 3: Teor de metais nos sedimentos lixiviado na baía do Guajará, coletados entre 2015 e 2016.

\begin{tabular}{|c|c|c|c|c|c|c|c|c|c|c|}
\hline \multirow{2}{*}{$\begin{array}{l}\text { Metais } \\
\mathrm{mg} \cdot \mathrm{Kg}^{-1}\end{array}$} & PT01 & & PT02 & & PT03 & & PT04 & & TEL & \multirow{2}{*}{$\begin{array}{l}\text { PEL e } \\
\text { CONAMA no 454/12 }\end{array}$} \\
\hline & $\mathrm{CH}$ & $\mathrm{MCH}$ & $\mathrm{CH}$ & $\mathrm{MCH}$ & $\mathrm{CH}$ & $\mathrm{MCH}$ & $\mathrm{CH}$ & $\mathrm{MCH}$ & & \\
\hline Cd & 0,8 & 0,7 & 1,1 & 0,8 & 0,8 & 0,8 & 0,6 & 0,1 & 0,6 & 3,5 \\
\hline $\mathrm{Cr}$ & 2,3 & 2,0 & 2,9 & 2,4 & 2,1 & 2,3 & 2,2 & 1,0 & 37,3 & 90,0 \\
\hline $\mathrm{Cu}$ & 5,9 & 4,9 & 6,8 & 14,7 & 7,2 & 8,0 & 4,8 & 1,2 & 35,7 & 197 \\
\hline $\mathrm{Fe}$ & 5878,8 & 5302,9 & 7357,9 & 5936,1 & 5874,0 & 6260,2 & 4891,2 & 950,6 & - & - \\
\hline
\end{tabular}

A fim de saber a biodisponibilidade dos metais no sedimento, foi realizado o lixiviamento das amostras, apresentando os resultados na tabela 3. Para obter informações sobre as fontes e grau de contaminação, realizaram-se os cálculos do Fator de Contaminação (FC), Índice de Geoacumulação (Igeo) e Fator de enriquecimento (FE). Utilizamos o Al para fazer a normalização por regressão, a fim de obter os valores de background para cada metal do estudo (figuras 11, 12, 13 e 14). Na Figura 15 e 16, apresentamos os valores do FC para cada metal em estudo. Tal fator é utilizado para quantificar o grau de contaminação que o ambiente estar sofrendo. Na tabela 4 são apresentados os resultados obtidos através do cálculo do

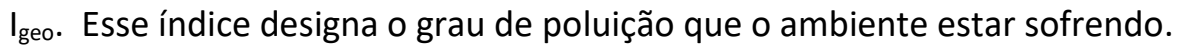

Tabela 4: Valores do Igeo encontrados na baía do Guajará, entre o período 2015 e 2016.

\begin{tabular}{|l|l|l|l|}
\hline Pontos & Cd & $\mathrm{Cr}$ & $\mathrm{Cu}$ \\
\hline PT01 $\mathbf{C H}$ & 0,26 & 0,22 & 0,18 \\
\hline PT01 $\mathbf{M C H}$ & 0,22 & 0,19 & 0,21 \\
\hline PT02 $\mathbf{C H}$ & 0,33 & 0,27 & 0,22 \\
\hline PT02 $\mathbf{M C H}$ & 0,26 & 0,23 & 0,15 \\
\hline PT03 CH & 0,24 & 0,2 & 0,15 \\
\hline PT03 $\mathbf{M C H}$ & 0,25 & 0,21 & 0,45 \\
\hline
\end{tabular}




\begin{tabular}{|l|l|l|l|}
\hline PT04 CH & 0,19 & 0,21 & 0,25 \\
\hline PT04 MCH & 0,03 & 0,10 & 0,04 \\
\hline
\end{tabular}

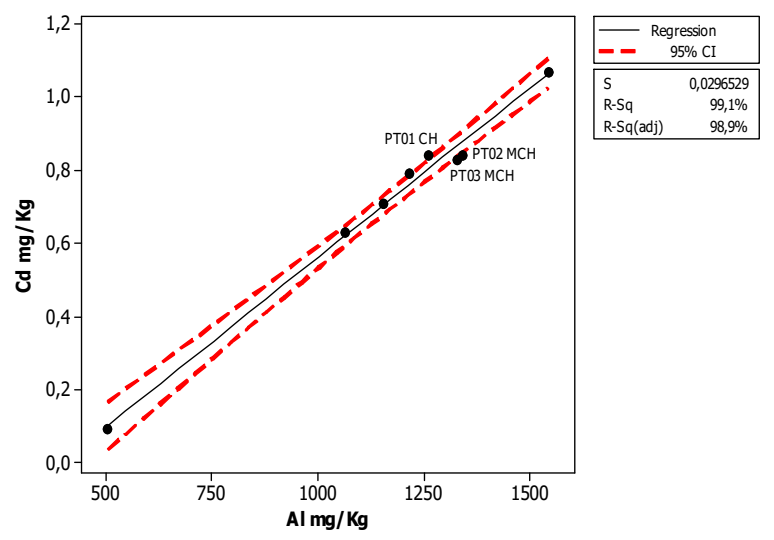

Figura 11: Relação entre a concentração do Cd versus o teor de Al.
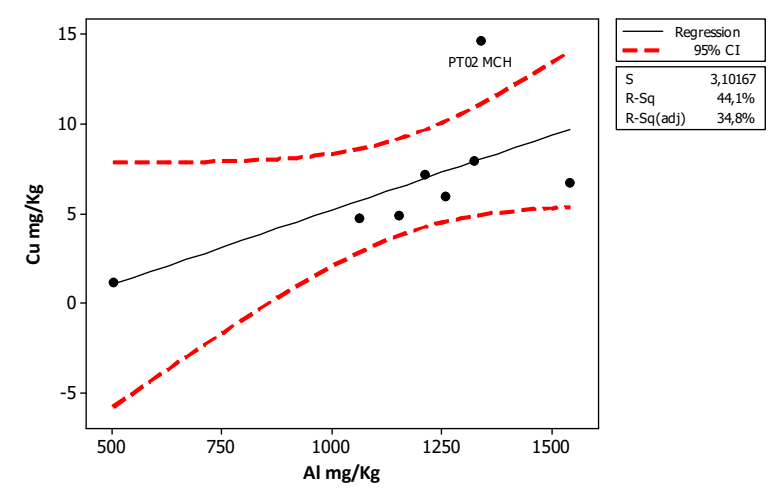

Figura 13: Relação entre a concentração do Cu versus o teor de Al.

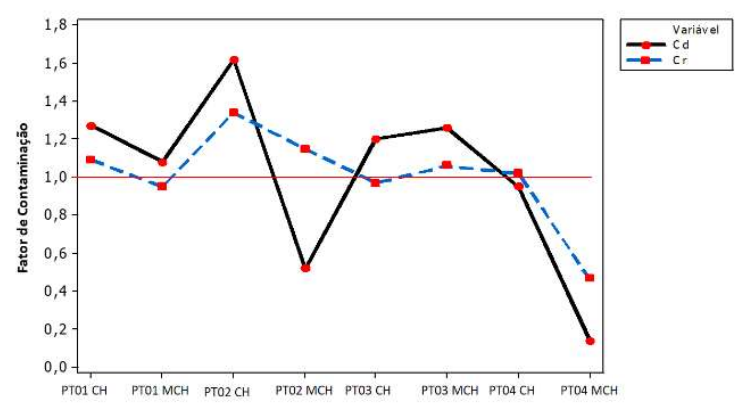

Figura 15: Valores dos FC do $\mathrm{Cd}$ e $\mathrm{Cr}$, calculados para os pontos de coletas na baía do Guajará, no período entre 2015 e 2016.

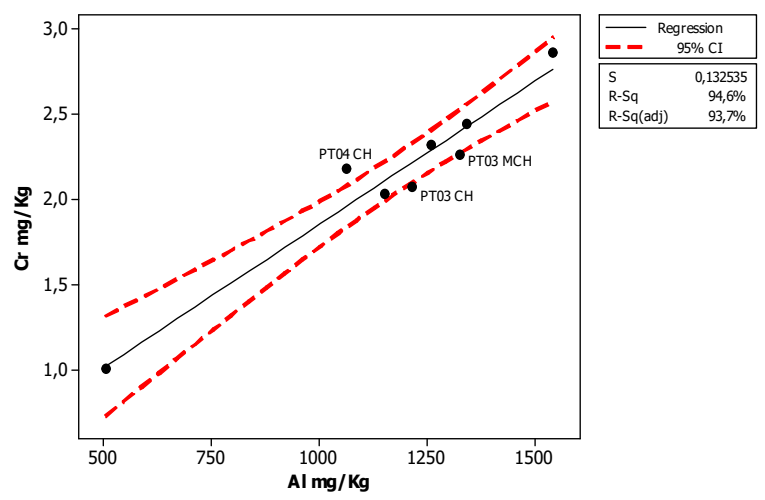

Figura 12: Relação entre a concentração do $\mathrm{Cr}$ versus o teor de Al.

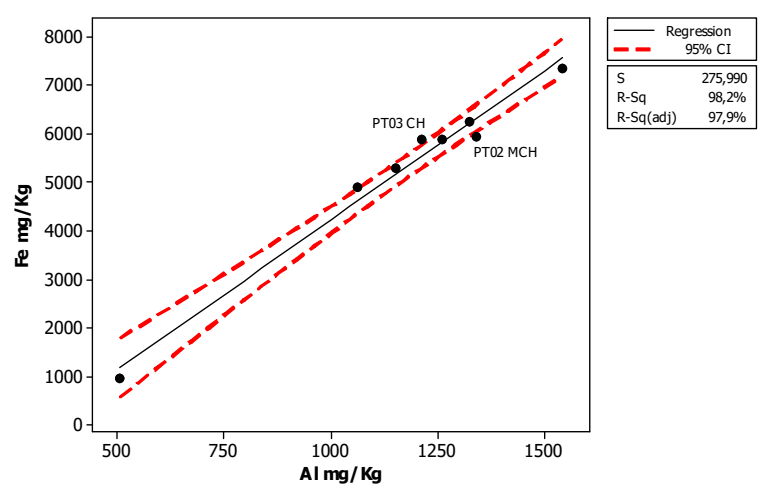

Figura 14: Relação entre a concentração do Fe versus o teor de Al.

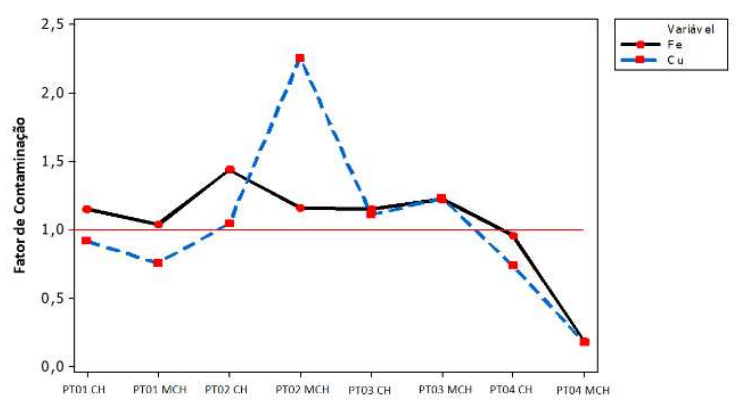

Figura 16: Valores dos FC do Fe e Cu, calculados para os pontos de coletas na baía do Guajará, no período entre 2015 e 2016.

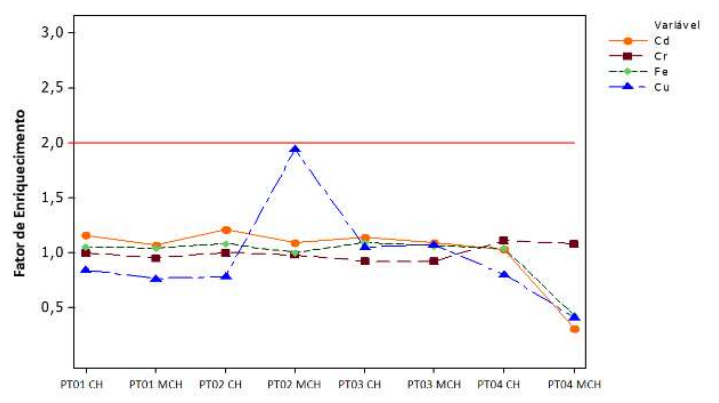

Figura 17: Valores de FE para o Cd, Cr, Fe e Cu, encontrado na baía do Guajará, entre o período 2015 e 2016. 


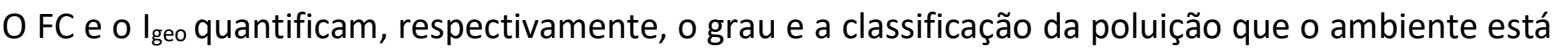
sofrendo. Entretanto, é necessário saber a origem dessa poluição, para tal informação realizou-se o cálculo do Fator de Enriquecimento (FE), em que designa se a poluição dos pontos em estudo é gerada através da atividade natural ou antropogênica. Os resultados do FE são apresentados na figura 17.

\section{DISCUSSÃO}

Ao analisar a concentrações dos metais no sedimento total (figuras 7, 8, 9 e 10), podemos observar que o $\mathrm{Cd}$ em todos os pontos, com exceção do PT01 do período menos chuvoso, apresentou concentrações que ficaram acima do permitido pela Resolução CONAMA no454/2012 e PEL. Com isso, chama-se atenção para tal investigação desse metal, pois o mesmo pode estar acarretando prejuízos ambientais que compromete a qualidade da água e da biota aquática, já que os organismos aquáticos apresentam dois tipos de comportamento em relação aos metais pesados: eles podem ser sensíveis à ação tóxica de um determinado metal, ou então eles podem não ser sensíveis, porém bioacumulando o metal, incrementando seu efeito nocivo através dos níveis tróficos das cadeias alimentares, colocando em risco os indivíduos situados nos topos destas cadeias (MALDONADO et al., 2009).

Dessa forma, é de suma importância uma vigilância minuciosa nos pontos em estudos, já que as amostras de sedimentos analisadas estão em concentração de $\mathrm{Cd}$ elevada. Os demais metais encontrados no sedimento total estão dentro do permitido pela CONAMA nำ54/2012, TEL e PEL. Ao analisar a biodisponibilidade do sedimento através da sua lixiviação (tabela 3), podemos observar que todos os metais estão dentro do permitido por TEL, PEL e CONAMA no 454/12. Dessa forma, comparando os resultados do $\mathrm{Cd}$ do sedimento lixiviado e da análise do sedimento total, podemos perceber que esse metal está se concentrando em maiores quantidades no sedimento total e não está sendo biodisponibilizado para o meio.

Segundo Maldonado et al. (2009), a entrada de metais pesados é facilitada pela dispersão de materiais particulados no ambiente, pois tais elementos traço entram em contato com o solo ou o ar e são espontaneamente carregados para os corpos hídricos, no qual os mesmos podem ficar disponíveis ou armazenados no sedimento. Tal ocorrência está acontecendo nos pontos em estudos, pois nota-se que o Cd está se concentrado no sedimento, mas não está sendo disponibilizado para o meio.

Um dos papeis importante que o sedimento exerce sobre o monitoramento ambiental, é a de concentrar metais por mais tempo e em maiores quantidades que a coluna d'água, devido a água passar por um processo de escoamento. No sedimento, uma vez que o metal se deposita nele, com o passar do tempo ele tende a aumentar essa concentração, tornando novamente disponível em solução. Dessa forma, através do sedimento, poderemos ter respostas da contaminação em longo prazo e saber as fontes e o grau de contaminação que o ambiente estudo está passando (POMPÊO, 2013).

Para isso utiliza-se o Fator de contaminação ( $F C$ ), Índice de Geoacumulação (Igeo) e Fator de enriquecimento (FE). Ao analisar o FC para o $\mathrm{Cd}$ e $\mathrm{Cr}$ (figura 15), observa-se que para o $\mathrm{Cd}$ os pontos PT02 MCH, PT04 CH e PT04 MCH e para o $\mathrm{Cr}$ os pontos PT01 MCH, PT03 CH e PT04 MCH, apresentaram valores 
menor que 1, representando baixa contaminação na área em estudo, os demais pontos apresentaram valores que ficaram na faixa de 1 à 3 , representando moderada contaminação por esses metais.

Na figura 16, o FC apresentou baixa contaminação para o Fe nos pontos PT04 CH e PT04 MCH e para o $\mathrm{Cu}$ nos pontos PT01 $\mathrm{CH}$ e MCH, PT04 $\mathrm{CH}$ e $\mathrm{MCH}$, os demais pontos para esses metais, apresentaram moderada contaminação. Oliveira et al. (2015) em seus estudos na baía do Guajará, também encontraram valores do FC similar ao presente estudo, ao qual concluiu que a concentração dos metais no sedimento desse corpo hídrico, não está afetando a biota aquática.

Apesar do presente estudo diagnosticar o ambiente como baixa e moderada contaminação, é importante realizar uma análise contínua, haja vista que o sedimento tem capacidade de acumular metais por longo tempo e isso pode aumentar o nível de contaminação da baía do Guajará. O cálculo do Igeo (tabela 4) demostrou que todos os pontos apresentaram classe 1, que corresponde a classificação de ambiente não poluído à moderadamente poluído (Tabela 2). O FE (figura 17) constata que a fonte de enriquecimento que está atingindo os pontos em estudo é predominantemente de origem natural. Tal ocorrência é devido aos processos físicos e químicos, como o intemperismo e carreamento do solo e rochas (SIQUEIRA et al., 2006).

Contudo, é de suma importância o monitoramento contínuo da baía do Guajará, haja vista que os metais quando atingem concentrações elevadas causam prejuízos para biota aquática, e isso coloca em risco a saúde da população humana, considerando que o processo de contaminação pode começar no sedimento e alcançar os níveis mais altos da cadeia alimentar. Para o monitoramento contínuo, é importante a implantação de planos e projetos de gestão, a fim de buscar o controle, prevenção e a progressiva eliminação das possíveis fontes de contaminação e assim buscar um desenvolvimento sustentável da baía do Guajará e garantir o desenvolvimento socioambiental e econômico dessa região (BRASIL, 2012).

\section{CONCLUSÕES}

Características como a redisposição e o acúmulo de poluentes, classificam o sedimento como uma matriz de forte importância nos estudos de impactos ambientais, já que através dele, podemos obter a resposta da poluição, por um período maior do campo de estudo. Tornando-se uma ferramenta positiva para a área de análise apresentada nessa pesquisa.

Na análise da baía do Guajará, o Cd apresentou valores acima do permitido pela resolução CONAMA no 454/12 e valores de PEL. É importante ressaltar que a concentração desse metal no sedimento lixiviado ficou dentro do permitido, ou seja, com tais resultados, sabemos que esse elemento traço está se acumulando apenas no sedimento, entretanto, se as condições ambientais, como as variações dos parâmetros físico-químico (como condições redox e $\mathrm{pH}$ ) mudarem, a concentração do $\mathrm{Cd}$ pode ser biodisponibilizadas para o meio e provocar danos para o ecossistema aquático e podendo atingir a saúde humana, através do processo de biomagnificação, em que concentrações de metais presente no sedimento pode ser transferidas para a cadeia trófica. 


\section{REFERÊNCIAS}

ARAÚJO, J. A.; SOUZA, R. F.. Aporte antropogênico de metais pesados em sedimentos de corrente de áreas de lixão, urbanizadas e agrícola, em Parelhas-RN, região semiárida do Brasil. Geografia (Londrina), v.21, n.3, p.5-22, 2012.

BELO, A.; QUINÁIA, S. P.; PLETSCH, A. L.. Avaliação da contaminação de metais em sedimentos superficiais das praias do lago de Itaipu. Química Nova, v.33, n.3, p.613-617, 2010.

BRASIL. Ministério do Meio Ambiente. Resolução CONAMA no454. Brasília, 2012.

CCME. Canadian Council of Ministers of the Environmet. Protocol for the derivation of Canadian Sediment quality guidelines for the protection of aquatic life. Ottawa, 1995.

COTTA, J. A. O.; REZENDE, M. O. O.; PIOVANI, M. R.. Avaliação Do Teor De Metais Em Sedimento Do Rio Betari No Parque Estadual Turístico Do Alto Ribeira - Petar, São Paulo, Brasil. Química Nova, v.29, n.1, p.40-45, 2006.

ELEFTHERIOU, A.; MCINTYRE, A.. Methods for the study of marine benthos. Blackwell Science Ltda, 2005.

FURLAN, N.; CALIJURI, M. C.; CUNHA, C. A. G.. Qualidade da Água e do Sedimento Avaliada a Partir da Concentração de Nutrientes Totais. Pesquisa e Tecnologia Minerva, v.6, n.1, p.91-98, 2009.

GREGÓRIO, A. M. S.; MENDES, A. C.. Batimetria e sedimentologia da baía de Guajará, Belém, estado do Pará. Amazônia: Ciência \& Desenvolvimento, Belém, v.5, n.9, p.53-72, 2009.

HAKANSON, L.. An Ecological Risk Index Aquatic Pollution Control. A sedimentological Aproach. Water Res., v.14, n.8, p.975-1001, 1980.

HORTELLANI, M. A.; SARKIS, J. E. S.; ABESSA; D. M. S.; SOUSA, E. C. P. M.. Avaliação da contaminação por elementos metálicos dos sedimentos do estuário Santos - São Vicente. Química Nova, v.31, n.1, p.10-19, 2008.

LICHT, O. A. B.. Prospecção Geoquímica: Princípios, Técnicas e Métodos. Rio de Janeiro: CPRM, 1998.

MALDONADO, A. C. D.; WENDLING, B.. Manejo de Ecossistemas Aquáticos Contaminados por Metais Pesados. Agropecuária Técnica, Paraíba, v.30, n.1, p.21-32, 2009.

MIGUENS, A. P.. Navegação a ciência e a arte. In: MIGUENS, A. P.. Navegação astronômica e derrotas: Manuais, Projetos, Pesquisas de Atualidades. 2 ed. 1996. p.47.

MIRANDA, A. G. O.; MENDES, A. C.. Evolução Batimétrica da Baía de Guajará, Belém/Pa. In: CONGRESSO DA ASSOCIAÇÃO BRASILEIRA DE ESTUDOS DO QUATERNÁRIO - ABEQUA, 11. Anais. 2007.

MOREIRA, R. C. A.; BOAVENTURA, G. R.. Referência geoquímica regional para a interpretação das concentrações de elementos químicos nos sedimentos da bacia do Lago Paranoá/DF. Química Nova, São Paulo, v.26, n.6, 2003.

MULLER, G.. Heavy metals in the sediment of the Rhine Changes seity. Umsch Wiss Tech, v.79, p.778-783, 1979.

OLIVEIRA, E. C.; LAFON, J. M.; MARTINS, J. A. C.; CARVALHO, J. H. S.; DIAS, F. F.; TADDEI, M. H. T.. Distribuição dos metais traços em sedimentos de fundo do sistema hidrográfico da região de Belém, PA (margem oeste da baía do Guajará e rio Carnapijó). Geochimica Brasiliensis, v.29, n.2, p.139-153, 2015.

POMPÊO, M.; PADIAL, P. R.; MARIANI, C. F.; SILVA S. C.; CARLOS, V. M.; SILVA, D. C. V. R.; PAIVA, T. C. B.; BRANDIMARTE, A. L.. Biodisponibilidade de metais no sedimento de um reservatório tropical urbano (reservatório Guarapiranga -São Paulo (SP), Brasil): há toxicidade potencial e heterogeneidade espacial?. Geochimica Brasiliensis, v.27, n.2, p.104-119, 2013.

REPULA, C. M. M.; CAMPOS, B. K.; GANZAROLLI, E. M.; LOPES, M. C.; QUINÁIA, P. Q.. Biomonitoramento de Cr e Pb em peixes de água doce. Química Nova, São Paulo, v.35, n.5, p.905-909, 2012.

SANTOS, A. L. F.; BORGES, L. O. S.; BOAVENTURA, G. R.. Indicator of the quality of sediments Piancó river, AnápolisGo and their environment implications. Scientia Plena, v.8, n.10, 2012

SILVA, W. L.; MATOS, R. H. R.; KRISTOSCH, G. C.. Geoquímica e Índice de Geoacumulação de Mercúrio em Sedimentos de Superfície do Estuário de Santos - Cubatão (SP). Química Nova, v.25, n.5, p.753-756, 2002.

SILVA, M. S. G. M.; BAPTISTA, D. F.; NESSIMIAN, J. L.; BUSS, D. F.; EGLER, M.. Estudo de caso: a comunidade de macro invertebrados aquáticos e sua utilização na avaliação da qualidade de água na bacia do rio Macaé, Estado do Rio de Janeiro. In: QUEIROZ, J. F.; SILVA, M. S. G. M.; TRIVINHOSTRIXINO, S.. Organismos bentônicos: biomonitoramento de qualidade de água. Jaguariúna: Embrapa, Meio Ambiente, 2008.

SIQUEIRA, G. W.; PEREIRA, S. F. P.; APRILE, F. M.. Determinação dos elementos-traço (Zn, Co e Ni) em sedimentos da Plataforma Continental Amazônica sob influência da descarga do rio Amazonas. Acta Amazônica, v.36, n.3, p.321-326, 2006.

TRINDADE, W. M.; HORN, A. H.; RIBEIRO, E. V.. Concentrações de Metais Pesados em sedimentos do Rio São Francisco entre Três Marias e Pirapora-Mg: Geoquímica e Classificação De Risco Ambiental. Geonomos, v.20, n.1, p.64-75, 2012.

YONGMING, H.; PEIXUAN, D.; JUNJI, C.; POSMENTIER, E. C.. Multivariate analysis of heavy metal contamination in urban dusts of Xi' an, Central China. Science of the Total Environment, Amsterdam, v.355, n.1-3, p.176-186, 2006. DOI: https://doi.org/10.1016/j.scitotenv.2005.02.026

A CBPC - Companhia Brasileira de Produção Científica (CNPJ: 11.221.422/0001-03) detém os direitos materiais desta publicação. Os direitos referem-se à publicação do trabalho em qualquer parte do mundo, incluindo os direitos às renovações, expansões e disseminações da contribuição, bem como outros direitos subsidiários. Todos os trabalhos publicados eletronicamente poderão posteriormente ser publicados em coletâneas impressas sob coordenação da Sustenere Publishing, da Companhia Brasileira de Produção Científica e seus parceiros autorizados. Os (as) autores (as) preservam os direitos autorais, mas não têm permissão para a publicação da contribuição em outro meio, impresso ou digital, em português ou em tradução. 